\title{
Single Pixel Compressive Camera for Fast Video Acquisition using Spatial Cluster Regularization
}

\author{
Yang Peng ${ }^{1}$ Yu Liu ${ }^{1 *}$, Kuiyan Lu' ${ }^{2}$ and Maojun Zhang ${ }^{1}$ \\ ${ }^{1}$ Department of System Engineering, National University of Defense Technology \\ Changsha,410073, China. \\ [e-mail: pengyang07@163.com, jasonyuliu@hotmail.com, zmj@upixels.com] \\ ${ }^{2}$ Shijiazhuang Flying College of PLAAF \\ Shijiazhuang, 050081, China. \\ *Corresponding author: Yu Liu
}

Received March 31, 2016; revised July 11, 2016; revised November 18, 2017; revised April 19, 2018; revised June 9, 2018; accepted June 21, 2018; published November 30, 2018

\begin{abstract}
Single pixel imaging technology has developed for years, however the video acquisition on the single pixel camera is not a well-studied problem in computer vision. This work proposes a new scheme for single pixel camera to acquire video data and a new regularization for robust signal recovery algorithm. The method establishes a single pixel video compressive sensing scheme to reconstruct the video clips in spatial domain by recovering the difference of the consecutive frames. Different from traditional data acquisition method works in transform domain, the proposed scheme reconstructs the video frames directly in spatial domain. At the same time, a new regularization called spatial cluster is introduced to improve the performance of signal reconstruction. The regularization derives from the observation that the nonzero coefficients often tend to be clustered in the difference of the consecutive video frames. We implement an experiment platform to illustrate the effectiveness of the proposed algorithm. Numerous experiments show the well performance of video acquisition and frame reconstruction on single pixel camera.
\end{abstract}

Keywords: Single pixel camera, compressive sensing, fast video acquisition, spatial cluster regularization 


\section{Introduction}

The cost of a high-resolution sensors in the visible spectrum has fallen dramatically over the last decade. Unfortunately, the sensors of the invisible spectrum still remain pricey. The research of high resolution imaging beyond the visible spectrum with low-resolution sensors or even single pixel sensor is of great potential in both the science field and commercial community.

Compressive sensing (CS) [1, 2] is a new theory belongs to the area of computer vision[3-8]. It is very popular in recent years as for its widely application in compressive imaging[9]. The single pixel camera is one of the prominent applications and attracts many researches' attention [10]. Although the imaging of the single pixel camera develops, the techniques for single pixel video camera are still not fully exploited.

A straightforward way to implement a compressive sensing video acquisition scheme is to consider each frame in a video sequence as a stand-alone image, perform compressive measurements on them, store the measurements, and reconstruct each frame. This method need quantity of measurements even if just generate a low-rate video clip. At the same time, the classic methods to recover the frames, such as OMP [11], FISTA [12] and Bregman [13], are based on the iterative scheme, leading to a high time consumption.

In recent years, improvements have been made for these classic methods [14-16]. Figueiredo and Nowak proposed soft thresholding method $[17,18]$ under the expectation minimization framework. In [19, 20], the total variation regularization is utilized to replace the $\mathrm{L}_{1}$-norm. Osher et al. [21, 22] introduced Bregman iterative regularization and then extended to many fields [23, 24]. Yin et al. [25, 26] improve the Bregman method in time consumption and performance. Burger [27-29] introduced an inverse scale space method for solving $\mathrm{L}_{1}$-minimization problems. One characteristic of all these algorithms is that the reconstruction process is iterative.

For another hand, several multi-pixel extensions to the single pixel camera have been proposed recently, with the goal of increasing the measurement rate [30-34]. One characteristic of all these extensions is that the system needs relative high resolution sensors, this makes them inapplicable for single pixel camera imaging where high resolution sensor arrays are prohibitively expensive.

In this paper, an approach for fast video data acquisition on single pixel camera in spatial domain is proposed. The video generation process focuses on reconstructing the difference of consecutive frames. The approach keeps the measurement matrix constant in the linear measurement process, thus we can establish mathematical relation among consecutive frames based on compressive sensing theory. Then a new regularization called spatial cluster is introduced. The regularization consists of sparsity prior and cluster prior which can improve the performance of reconstruction algorithm. After reconstructing the difference of consecutive video frames, we can obtain the video by adding the recovered difference to the last frame one by one. In all, the new video acquisition scheme can decrease the data size and computational cost by reconstructing the difference of frames instead of video frames.

This paper is organized as follows. Section 2 introduces the description of the model of the single pixel camera scheme. Section 3 covers the new regularization and the reconstruction algorithm. In section 4 , the results in numerical simulations and real data are shown and we also compare our algorithm with several other methods. Lastly, we conclude with a short discussion in Section 5. 


\section{Imaging Model}

We implement the single pixel camera with a XGA 2xLVDS DMD from the Texas Instruments controlled by DMD Discovery 4100 chipest. The resolution of the DMD is $1920 \times 1080$ and each micro mirror can flutter at maximum rate of $20 \mathrm{kHz}$, which can produce an approximate 10fps video sequence with a resolution of $100 \times 100$ in theory. The architecture of the platform is shown in Fig. 1. The scene is lighted by the modulated light from DMD, the binary coded patterns are recorded as $A$. An electric appliance is adopted to make the scene moving around. The Newport single pixel sensor 818-UV/DB obtains the measurement $b$ synchronized. Each frame $x$ of the video can be reconstructed based on compressive sensing theory.

$$
b=A x
$$

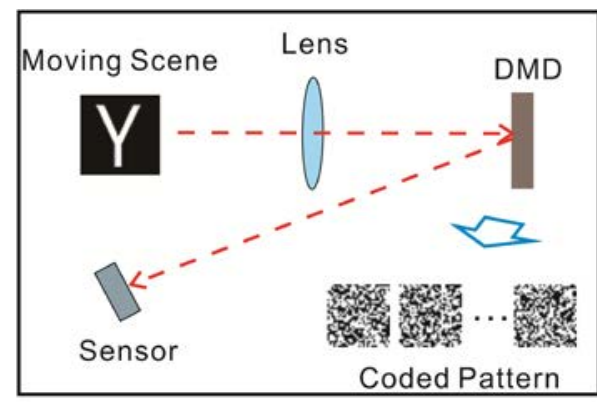

(a)

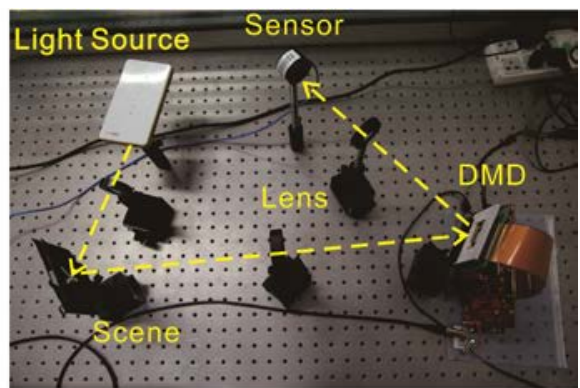

(b)

Fig. 1. (a) Schematic of our design and a photograph of our prototype; (b) Photograph of our platform. An electric appliance is adopted to make the scene moving around.

To improve the video acquisition on single pixel imaging system, a new imaging model is proposed. This model refers to recovering the difference of the consecutive frames rather than directly obtaining the video frame one by one. As the difference of the consecutive frames is sparse in spatial domain, we can recover the signal directly. For another hand, the number of measurement decreases as most pixel in difference is zero.

The detailed description of the video acquisition model is shown in Fig. 2. The testing frames are employed from datasets CDnet[35] donated as Backdoor. These frames represent a girl passing through the backdoor. The crucial element of the acquisition scheme is to fix the measurement matrix $A$ in Eq.1. This indicates that we shall use the same set of binary coded patterns cyclically. The initial frame $x_{1}$ is recovered on the framework of compressive sensing theroy. For the upcoming video frames, we refer to recovering the difference of the consecutive frames rather than directly obtaining the video frame. As the measurement matrix is fixed, we can establish the mathematical relation between the difference of consecutive frames and the measurement as below:

$$
\nabla b=b_{n}-b_{n-1}=A x_{n}-A x_{n-1}=A\left(x_{n}-x_{n-1}\right)
$$

Where $b_{n}$ and $b_{n-1}$ are two neighboring measurements, $A$ is the constant measurement matrix, $x_{n}$ and $x_{n-1}$ are the consecutive frames. From Eq.2, we can find that if the differene satisfies sparsity prior, the signal can be reconstucted in the framework of compressive sensing theory. 


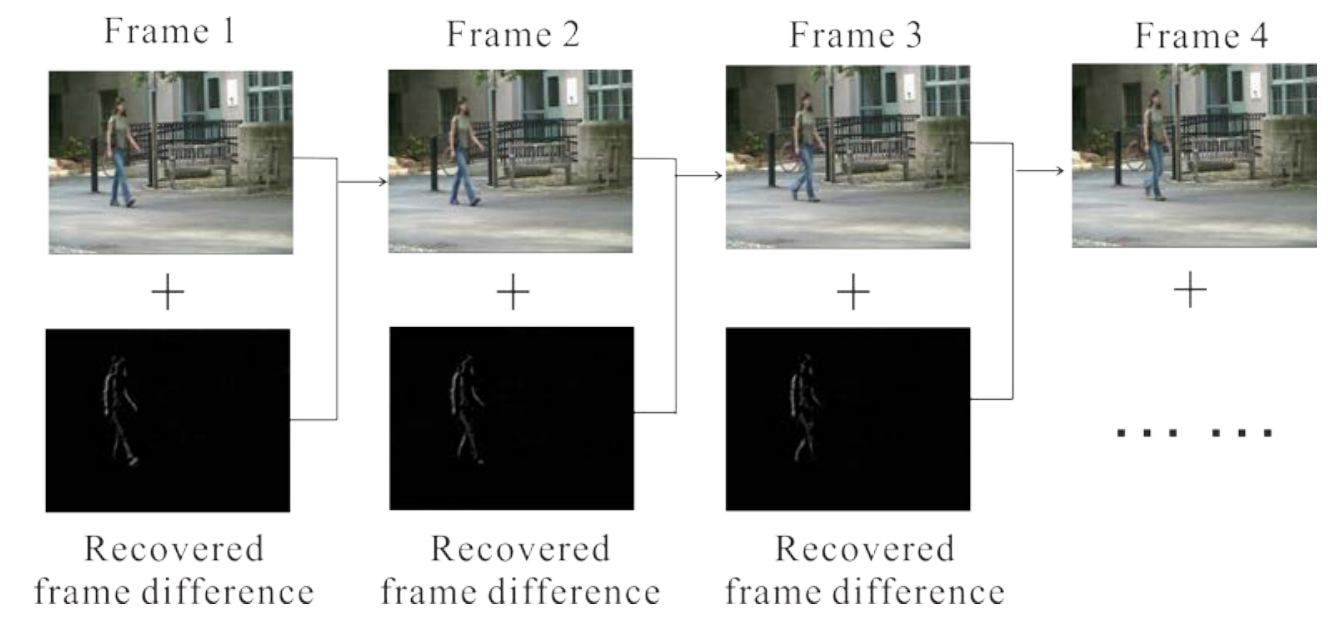

Fig. 2. The description of the compressive sensing video scheme. The testing frames are employed from datasets CDnet donated as Backdoor. These frames represent a girl passing through the backdoor.

Our experiment platform is a compressive imaging system based on the theory of compressive sensing. The new video acquisition scheme is adopted to improve the efficiency of the video generation on single pixel imaging system. At the same time, the single pixel sensor can response to both the visible spectrum and invisible spectrum.

\section{Spatial Cluster Prior}

When analyzing the difference of video frames of natural scene, we find that the nonzero elements always have the attribute of spatial sparsity and cluster. If these prior can be fully utilized, the performance of the signal reconstruction can be improved. In this section, a new regularization combines the sparsity and the spatial cluster is proposed. Then the new regularization based signal reconstruction algorithm which can enable a better performance is introduced.

\subsection{Spatial Cluster Regularization}

By observing and analyzing natural scene video data, two important properties of the difference of the consecutive frames are figured out: sparsity and cluster. In the following, a further analysis of these two properties are illustrated by an example selected from stationary video data. Fig. 3 shows the instance of real video frames of a letter ' $Y$ '. We can find that in Fig. 3(c), the changes in difference image cluster in spatial domain. In stationary video, changes occur while objects moving, and the changes are always small. From the intensity distribution of the difference image in Fig. 3(d), we can figure out the sparsity prior as well. In all, the nonzero elements in difference of the frames are sparse and always appear cluster but not randomly distributed. More specially, the R, G and B channel share the same properties in each channel respectively.

By enforcing these two properties, it leads to several advantages: 1) Accelerating the convergence. As the new regularization is added, the algorithm has a more rapid convergence. 2) Decreasing the minimal number of measurements. The rapid convergence leads to less measurements. 3) Improving robustness to noise and preventing the recovered data from 
having artifacts. These advantages enable the proposed algorithm to efficiently obtain stable and robust sparse reconstruction.

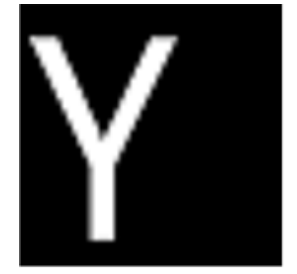

(a)

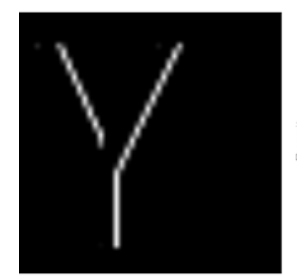

(c)

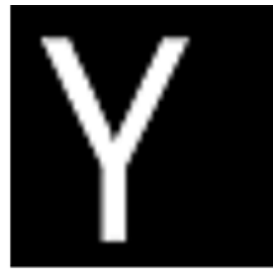

(b)

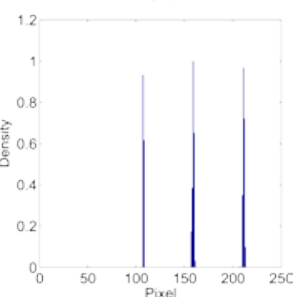

(d)

Fig. 3. An analysis of the real frame data: (a) and (b) are two consecutive video frames, (c) the difference image of the two frames, (d) the intensity distribution of the difference image.

Therefore these properties can be used as a new regularization in the optimization process to improve the performance. In this paper, a cluster operator is introduced to depict the property of cluster in mathematical form. The operator is to measure the cluster and sparsity of the difference between two adjacent elements of the signal. For the clustered signal $x \in R^{n}$, the nonzero coefficients group in certain region and the $\mathrm{L}_{2}$-norm of the cluster operator on the difference signal tends to be small. This regularization is added to the objective function in the optimization process to improve the performance.

$$
\min \|\nabla x\|_{2}, \quad x \in R^{n}
$$

Where $\nabla(\bullet)$ represents the cluster operator.

\subsection{Spatial Cluster Regularization Based Algorithm (SCA)}

The combination of the sparsity prior and the cluster prior is utilized as a regularization in the signal reconstruction. The objective function of optimization problem changes to:

$$
\min \alpha\|x\|_{1}+\frac{\beta}{2}\|\nabla x\|_{2}^{2}+\frac{1}{2}\|A x-b\|_{2}^{2}
$$

Where $A \in R^{m \times n}$ is the measurement matrix, $x \in R^{n}$ is the spares signal, and $b \in R^{m}$ is the measurement vector, $\nabla(\bullet)$ represents the difference operator. $\alpha$ and $\beta$ are parameters used to balance the two prior terms.

For a 2D image signal, it is usually reshaped to a $1 \mathrm{D}$ vector to implement the reconstruction. For a reshaped signal $x \in R^{n}$, the cluster operator can be transform to a matrix multiplication as shown in Eq.5.

$$
\nabla x=T x
$$


Define matrix $T$ as transform matrix. We can easily obtain the specific value of the matrix $T$ by the definition of the difference operator.

$$
T=\left[\begin{array}{ccccc}
1 & -1 & & & \\
& 1 & -1 & & \\
& & \ddots & \ddots & \\
& & & 1 & -1 \\
& & & & 1
\end{array}\right]_{n \times n}
$$

The $\mathrm{L}_{2}$-norm of the $\nabla x$ can be represented as below:

$$
\|\nabla x\|_{2}=\|T x\|_{2}
$$

Then the Eq.4 is transformed to an unconstraint convex optimization:

$$
\underset{x}{\arg \min } \alpha\|x\|_{1}+\frac{\beta}{2}\|T x\|_{2}^{2}+\frac{1}{2}\|A x-b\|_{2}^{2}
$$

The $\mathrm{L}_{2}$-norm terms of the Eq.8 are approximated by their first order Taylor expansion at $x=x^{k}$ and an $\mathrm{L}_{2}$-proximity term at $x=x^{k}$.

$$
\begin{gathered}
\frac{1}{2}\|T x\|_{2}^{2}=\frac{1}{2}\left(T x^{k}\right)^{2}+T^{T} T x^{k} \cdot x+\frac{1}{2 \sigma}\left\|x-x^{k}\right\|_{2}^{2} \\
\frac{1}{2}\|A x-b\|_{2}^{2}=\frac{1}{2}\left(A x^{k}-b\right)^{2}+A^{T}\left(A x^{k}-b\right) \cdot x+\frac{1}{2 \omega}\left\|x-x^{k}\right\|_{2}^{2}
\end{gathered}
$$

Where $\sigma$ and $\omega$ are positive and sever as the error control parameters.

Replacing the $\mathrm{L}_{2}$-norm of Eq.8 with Eq.9, Eq.10 and discarding the constant terms, we can obtain a new objective function:

$$
\underset{x}{\arg \min } \alpha\|x\|_{1}+\beta T^{T} T x^{k} \cdot x+A^{T}\left(A x^{k}-b\right) \cdot x+\frac{1}{2 \delta}\left\|x-x^{k}\right\|_{2}^{2}
$$

Where $\delta$ is a constant relevant to parameters $\sigma$ and $\omega$. Finally we can yield a new iterative scheme:

$$
x^{k+1} \leftarrow \min _{x} \alpha\|x\|_{1}+\frac{1}{2 \delta}\left\|x-\left(x^{k}-\left(\left(\beta T^{T} T+A^{T} A\right) x^{k}-A^{T} b\right)\right)\right\|_{2}^{2}
$$

Motivated by the Bregman iteration, we replace the $\mathrm{L}_{1}$-norm term by the Bregman distance based on a convex function $J(x)=\alpha\|x\|_{1}$ :

$$
x^{k+1} \leftarrow \min _{x} D_{J}^{p^{k}}\left(x, x^{k}\right)+H(x)
$$

Where

$$
H(x)=\frac{1}{2 \delta}\left\|x-\left(x^{k}-\left(\left(\beta T^{T} T+A^{T} A\right) x^{k}-A^{T} b\right)\right)\right\|_{2}^{2}
$$

To minimize the objective function Eq.13, we derivatives the function and yield the optimality conditions:

$$
0=p^{k+1}-p^{k}+\frac{1}{\delta}\left(x^{k+1}-\left(x^{k}-\left(\left(\beta T^{T} T+A^{T} A\right) x^{k}-A^{T} b\right)\right)\right)
$$

We obtain that: 


$$
p^{k+1}=p^{k}-\frac{1}{\delta}\left(x^{k+1}-x^{k}\right)+A^{T} b-\left(\beta T^{T} T+A^{T} A\right) x^{k}
$$

Letting that $p^{0}=0$ and $x^{0}=0$, then the sequence of $p^{k}$ can be calculated one by one till $k=0$ and finally we can yield:

$$
p^{k+1}=\frac{1}{\delta} \sum_{j=0}^{k}\left(A^{T} b-\left(\beta T^{T} T+A^{T} A\right) x^{k}\right)-\frac{x^{k+1}}{\delta}
$$

Letting:

$$
u^{k}=\frac{1}{\delta} \sum_{j=0}^{k}\left(A^{T} b-\left(\beta T^{T} T+A^{T} A\right) x^{k}\right)
$$

The optimality conditions of the $x^{k+1}$ can be obtained by the shrinkage operation, which is also referred to as soft thresholding:

$$
x^{k+1} \leftarrow \delta \cdot \operatorname{shrink}\left(u^{k}, \alpha\right)
$$

Where $y, \alpha \in R$, the shrinkage operator is defined:

$$
\operatorname{shrink}(y, \alpha)=\operatorname{sgn}(y) \max (|y|-\alpha, 0)
$$

This is the SCA method and its concise description is shown in Algorithm 1. This algorithm is an extremely fast algorithm. At the same time, it is very simple to program, involving only matrix multiplication and scalar shrinkage.

Algorithm 1 Spatial Cluster Algorithm (SCA)

Initialize: $x^{0}=0, u^{0}=0, \quad k=0$

While not converge do

$$
\begin{aligned}
& u^{k+1} \leftarrow u^{k}+A^{T} b-\left(\beta T^{T} T+A^{T} A\right) x^{k} \\
& x^{k+1} \leftarrow \delta \cdot \operatorname{shrink}\left(u^{k+1}, \alpha\right) \\
& k=k+1
\end{aligned}
$$

\section{Experiments and Analysis}

To demonstrate the advantage of our method, the experiments are done both on the 1D simulated signal and image data obtained from the CDnet datasets[35]. At the same time, we compare the performance of proposed method with state-of-the-art methods including SPGL1-LASSO [15], FISTA [12], Accelerated Linearized Bregman (ALB) [26], OMP[11], Inverse Scale Space method (ISS) [29], and Hard Thresholding Pursuit(HTP)[36]. In order to make a quantitative comparison and evaluation of these algorithms, the recovery error which is defined as the relative difference between the recovery signal and the ground truth signal is adopted to the 1D signal, the structural similarity index SSIM, as proposed in [37], is used to evaluate the quality of image reconstruction additionally. All experiments are done on the matlab environment with a $2.0 \mathrm{GHz}$ laptop. 


\subsection{D Simulated Signals}

We randomly generate a sparse $1 \mathrm{D}$ signal with values 1 and 0 . The length of the signal is set to 200. The number of nonzero elements is set to 10 . The nonzero elements cluster into two groups. The measurement matrix are randomly distributed binary patterns with a one-to-zero ratio of 1:1. Zero-mean Gaussian noise with standard deviation $\sigma=0.1$ is added to the measurements. Fig. 4 shows one generated signal and its recovered results by different algorithms with the measurement number of 30 . As the measurement number is only 3 times of the number of nonzero elements, all algorithms cannot obtain a perfect recovery results, however our method obtains relatively sparsity results with the low running time.
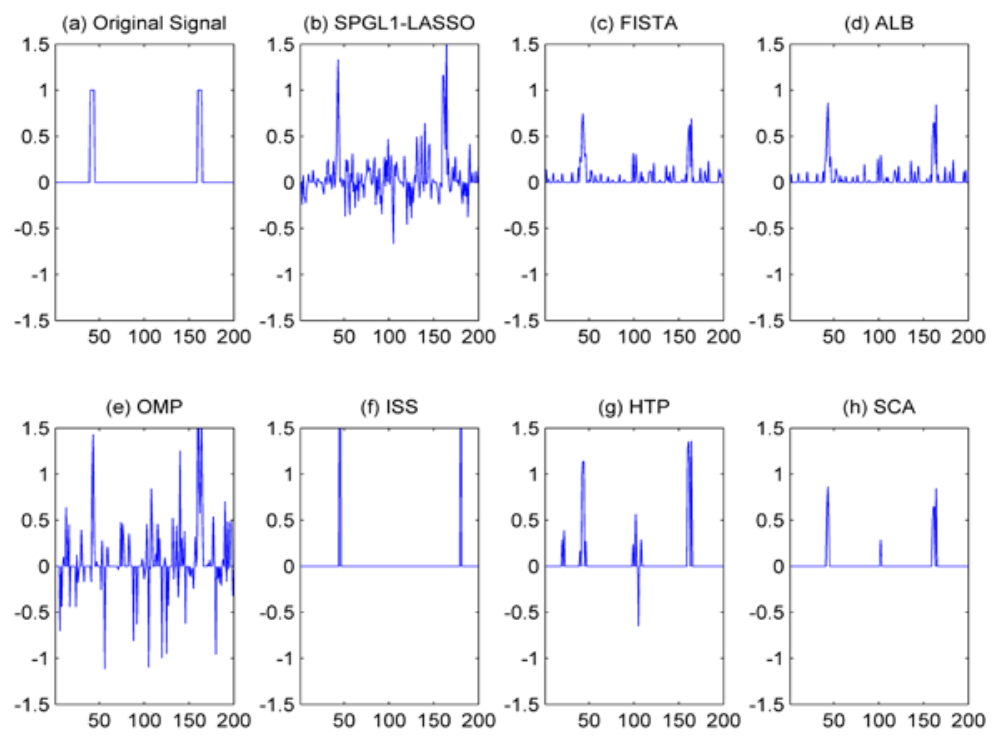

Fig. 4. Recovery results of 1D data with noise. (a) Original data; (b) SPGL1-Lasso (error is 0.9111 and time is 1.3433 seconds); (c) FISTA (error is 0.9312 and time is 0.0209 seconds); (d) ALB (error is 0.5960 and time is 0.0269 seconds); (e) OMP (error is 1.4515 and time is 0.1813 seconds); (f) ISS (error is 2.224 and time is 0.0497 seconds); (e) ISS (error is 1.8352 and time is 0.0374 seconds); (h)SCA recovery (error is 0.8211 and time is 0.0345 seconds)

To study how the measurement number works on the performance of algorithms, we change the measurements number and record the recovery results respectively. We repeat the experiment 50 times for each of the measurement numbers. Fig. 5 shows the performance of the algorithms with increasing measurements in terms of the recovery error and running time. Fig. 5(a) shows the average performance of 7 algorithms with increasing measurements in terms of reconstruction error. $\mathrm{X}$-axis denotes the measurement number and $\mathrm{y}$-axis denotes the reconstruction error. We can see that SCA has a lowest reconstruction error in almost every measurement. At the same time, SCA method is more stable as it acquires nearly the same reconstruction error in 50 experiments. The computational cost curve of the 7 algorithms is plotted in Fig. 5(b). Numerical values on the x-axis denote the measurement number while those on the y-axis denote the running time. The running time of SCA also is lowest for all measurements. Overall, SCA method obtains the best and stable performance with low computation. 

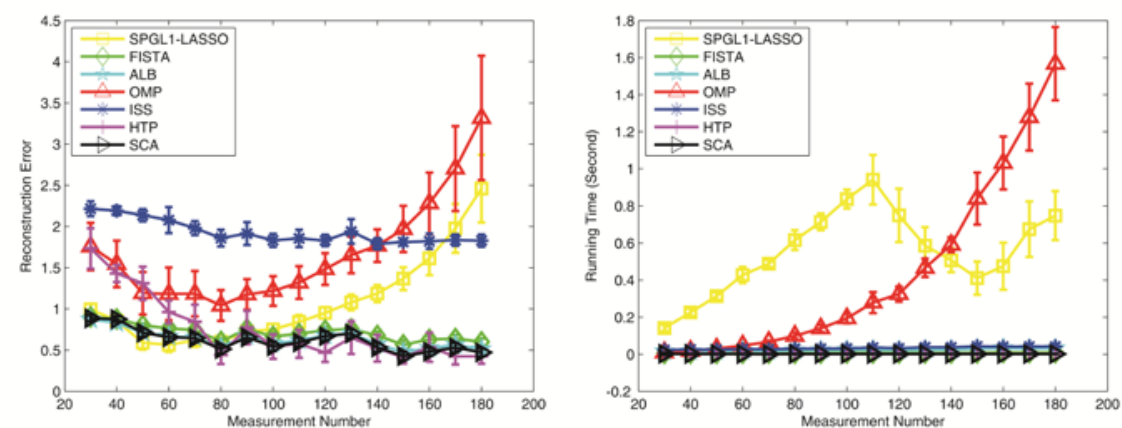

Fig. 5. Performance curve. (a) Reconstruction errors; (b) Running times.

\subsection{D Signals}

We also test our proposed method with different algorithms by using both simulated and real video sequences. The frame size is limited to $30 \times 30$ pixels for all video sequences. This limitation is simply due to the operational capability of our hardware. The structural similarity index (SSIM) is used to evaluate the reconstruction quality. The value of the SSIM ranges from 0 to 1 , and the bigger the better. SSIM takes the degradation of the structural information in the image into consideration, and therefore can provide a better and more comprehensive evaluation than traditional criterion such as the peak signal-to-noise ratio PSNR, which assesses the reconstruction qualities solely based on the intensity differences between the reconstructed and original frames.

The video sequences are composed of computer-generated frames and real scene sequences employed from CDnet dataset. Each computer-generated sequence has 15 frames per second, showing a rectangular white box moving from left to right on a fixed background scene. By choosing different box sizes and moving speeds, four kinds of simulated video sequences are generated. Another 8 test video sequences are obtained from CDnet dataset which include different types of periodical or irregular background motion. A total of 12 video sequences are used in this study to evaluate the algorithms. A few example of computer-generated video frames are shown in Fig. 6.The measurement number is set to the $30 \%$ of the pixel number. Zero-mean Gaussian noise with standard deviation $\sigma=0.1$ is added to the measurements.

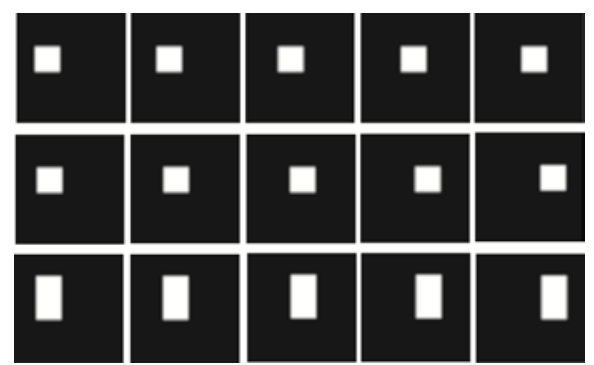

Fig. 6. An example of computer-generated video sequences. Each row represents five frames in one simulated video sequence.

An example of reconstructed frames is shown in Fig. 7. The first row shows the second frame of the simulated video sequences. The second row shows the 13th frame of the sequences. Fig. 7(a) illustrates the ground truth video data. Fig. 7(b)-(f) present video frames reconstructed with different methods. 


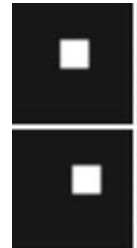

(a)

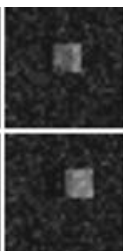

(b)

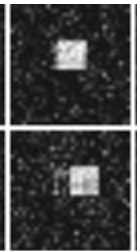

(c)

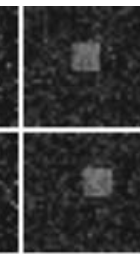

(d)

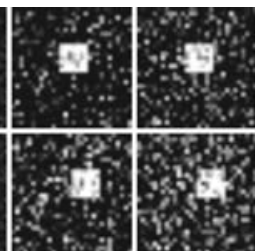

(e)

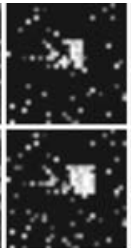

(g)

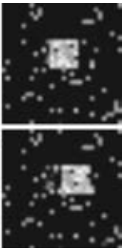

(h)

Fig. 7. An example of reconstructed frames from stimulated video sequencewith different methods. (a) Ground truth; (b) SPGL1-Lasso; (c) FISTA; (d) ALB; (e) OMP; (f)ISS; (g)HTP; (h) SCA.

For the reconstructed video sequences, we observe that the SPGL1-Lasso, method has an acceptable result with high expense in time consumption. The FISTA, ALB, OMP, HTP and ISS method can only obtain a skeleton of the object. The proposed SCA method can reconstruct a satisfied result. The SSIM values of these different methods with different measurement number are depicted in Fig. 8. From the figure, we can see that the SCA method has the best performance in all experiments.

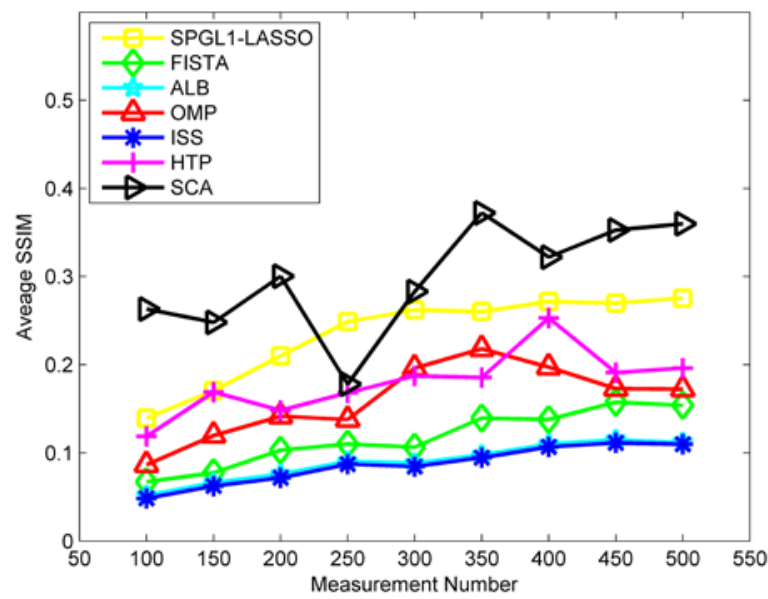

Fig. 8. The average SSIM values of different methods with different measurement number on simulated video sequence

The SSIM values of these different methods on all the datasets are summarized in Table 1. For the simulated video sequences, we observe that the performance of the SCA method has the best reconstruction quality in most video sequence. The last 4 video sequence has a higher SSIM value than other data because the speed of moving object is relatively slow. The difference of the consecutive frames is more sparse, leading to a better performance of reconstruction algorithms.

Table 1. Video reconstruction results of 7 methods for simulated video sequences.

\begin{tabular}{|l|c|c|c|c|c|c|c|}
\hline \multirow{2}{*}{\multicolumn{1}{c|}{ Video }} & \multicolumn{7}{|c|}{ Average SSIM } \\
\cline { 2 - 8 } & SPGL1-Lasso & FISTA & ALB & OMP & ISS & HTP & SCA \\
\hline computer-generated-1 & 0.154 & 0.101 & 0.224 & 0.098 & 0.113 & 0.146 & 0.351 \\
\hline computer-generated-2 & 0.146 & 0.133 & 0.208 & 0.102 & 0.122 & 0.144 & 0.305 \\
\hline
\end{tabular}




\begin{tabular}{|c|c|c|c|c|c|c|c|}
\hline computer-generated-3 & 0.159 & 0.122 & 0.312 & 0.114 & 0.109 & 0.151 & 0.310 \\
\hline computer-generated-4 & 0.136 & 0.115 & 0.217 & 0.109 & 0.106 & 0.129 & 0.360 \\
\hline Backdoor & 0.158 & 0.105 & 0.221 & 0.111 & 0.114 & 0.153 & 0.355 \\
\hline BusStation & 0.149 & 0.102 & 0.230 & 0.108 & 0.110 & 0.145 & 0.353 \\
\hline FluidHighway & 0.151 & 0.111 & 0.225 & 0.113 & 0.115 & 0.160 & 0.350 \\
\hline Pedestrians & 0.143 & 0.108 & 0.236 & 0.099 & 0.107 & 0.122 & 0.362 \\
\hline Skating & 0.271 & 0.243 & 0.441 & 0.199 & 0.249 & 0.251 & 0.513 \\
\hline Traffic & 0.311 & 0.304 & 0.551 & 0.295 & 0.332 & 0.301 & 0.598 \\
\hline Turbulence0 & 0.307 & 0.318 & 0.591 & 0.301 & 0.341 & 0.298 & 0.587 \\
\hline WinterStreet & 0.365 & 0.370 & 0.621 & 0.316 & 0.401 & 0.359 & 0.712 \\
\hline
\end{tabular}

The same experiment is done on real video sequences. We use the single pixel imaging platform shown in Fig. 1 to acquire the video clips. The size of the video frame is set to $70 \times 70$. The measurement rate is set to $30 \%$ of the resolution. Limited to the operational capability of hardware, the speed of moving speed is set to approximate 3 pixels per second. We test all the method on different scene such as moving letters, moving boxes.

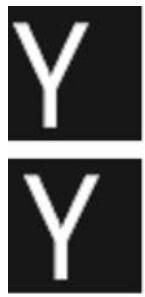

(a)
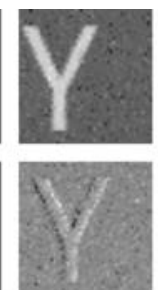

(b)

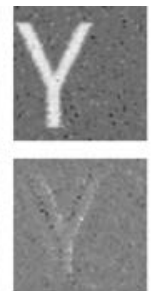

(c)
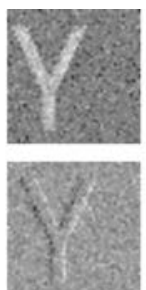

(d)

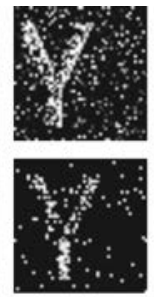

(e)
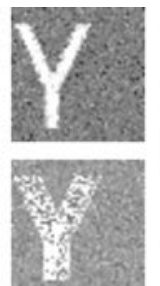

(f)

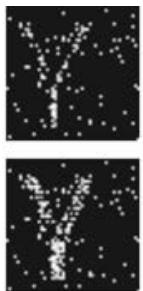

(g)
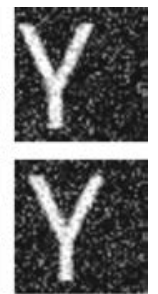

(h)

Fig. 9. An example of reconstructed frames of $70 \times 70$ from video sequence using the differencing method. (a) Ground truth; (b) SPGL1-Lasso; (c) FISTA; (d) ALB; (e) OMP; (f)ISS; (g)HTP; (h) SCA.

An example of reconstructed frames of a moving letter ' $\mathrm{Y}$ ' is shown in Fig. 9. The first row illustrates the second frame of the video sequence. The second row illustrates the sixth frame of the video sequence. To make a clear view of the reconstruction results, the image data are all normalized. Fig. 9(a) shows the ground truth video data. Fig. 9(b)-(h) present video frames reconstructed with different methods. From the results we can obtain an almost same conclusion with the stimulated video frames. The proposed SCA method has the best performance on the quality of reconstruction. We finally obtain a video clips based on the video acquisition in a fast time. The results are shown in Fig. 10. To show a visible results, the difference signals are all normalized. The results on both simulated and real video sequences show the proposed algorithms gain marked improvement over previous algorithms when cluster priors are available. 


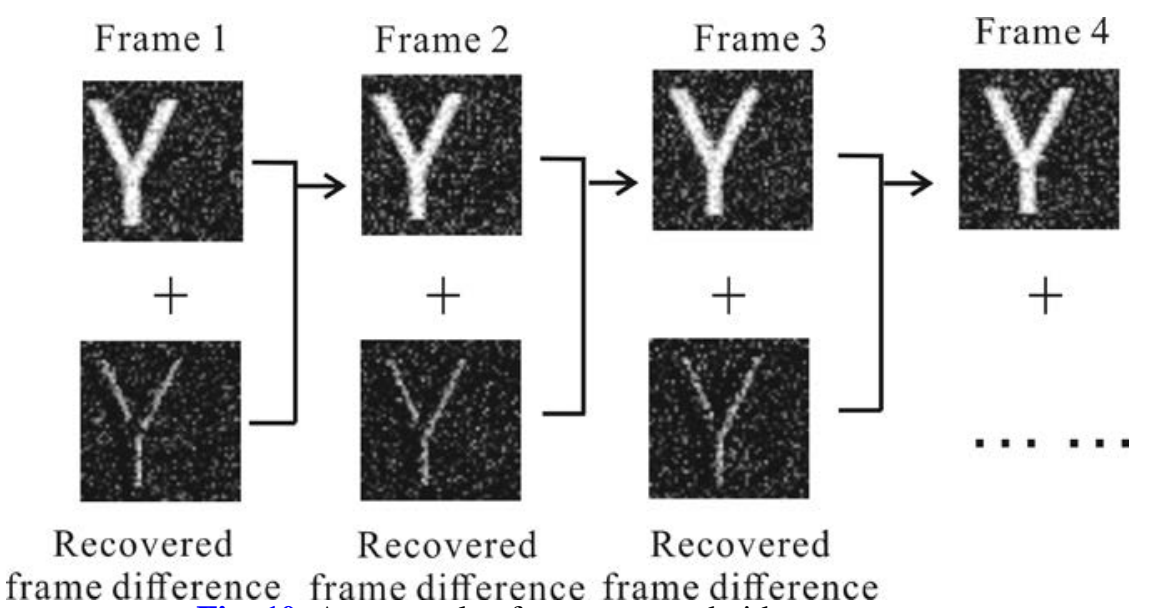

Fig. 10. An example of reconstructed video sequence

We also tested our proposed method on natural image signal recovery. In this experiment, signals of interest are natural images of size $64 \times 64$ such as the $64 \times 64$ Lena, House and Boat images. The sparse transform basis used for these natural images is the well-known Daubechies 9/7 wavelet transform. All images are implicitly regarded as 1-D signals of length 64×64. Fig. 11 shows the visually reconstructed $64 \times 64$ Lena image from $30 \%$ of measurements by using different algorithms. The OMP method is not employed because of its high complexity and bad performance. Fig. 11(a) is the ground truth video data. Fig. 11(b)-(g) present natural image reconstructed with different methods. From the experiments, we can see that our method has a relative low reconstruction error and it works well on 2D natural image signals.

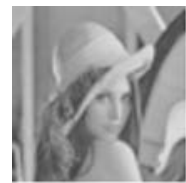

(a)

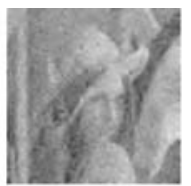

(b)

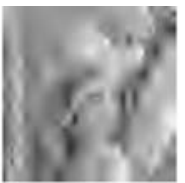

(c)

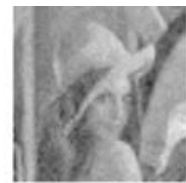

(d)

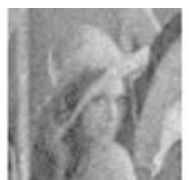

(e)

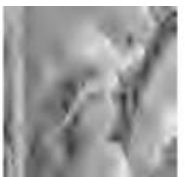

(f)

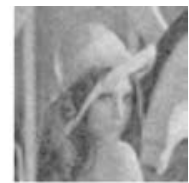

$(\mathrm{g})$

Fig. 11. An example of reconstructed natrual siganl. (a) Ground truth; (b) SPGL1-Lasso (error is 0.2318); (c) FISTA (error is 0.1668); (d) ALB (error is 0.1098); (e) ISS (error is 0.1132); (f) HTP (error is 0.1581 ); (g)SCA recovery (error is 0.0911 )

\section{Conclusion}

In this paper, a new model for video acquisition of the single pixel camera in spatial domain is introduced and a novel regularization called Spatial Cluster is proposed. It has been applied to sparse recovery on both simulated and practical data. Experimental results demonstrate the performance guarantee of the proposed algorithm and show marked improvement over previous algorithms. There also exists some limitations of the method. The system exploits the difference of the consecutive frames, therefore, the scene with extremely big changes cannot be handled. Since the measurement rate of the hardware, the video acquisition is not efficiency. In the future, we will use a low resolution sensor to replace the single pixel detector to improve the measurement rate. At the same time, the reconstruction method is also needed to be improved to real-time scale. 


\section{Acknowledgements}

This work was supported by the National Natural Science Foundation of China under Grant number 61403403.

\section{References}

[1] Donoho, D., “Compressed sensing, ” IEEE Transactions on Information Theory, vol.52 no.4, pp. 1289-1306, 2006.Article (CrossRef Link).

[2] Candes, E.J., Romberg, J., Tao, T., "Robust uncertainty principles: exact signal reconstruction from highly incomplete frequency information," IEEE Transactions on Information Theory, vol.52 no.2, pp. 489-509, 2006.Article (CrossRef Link).

[3] Ali, N., et al. "A Novel Image Retrieval Based on Visual Words Integration of SIFT and SURF," PLOS ONE, vol.11 no.6, 2016. Article (CrossRef Link).

[4] Ashraf, R., et al. "Content Based Image Retrieval Using Embedded Neural Networks with Bandletized Regions," Entropy, vol.17 no.6, pp: 3552-3580, 2015. Article (CrossRef Link).

[5] Ali, N., Bajwa, K.B., Sablatnig R., et al. "Image retrieval by addition of spatial information based on histograms of triangular regions," Computers \& Electrical Engineering, vol.54, pp.539-550, 2016. Article (CrossRef Link).

[6] Minghu, W., Xiuchang, Z. "Distributed Video Compressive Sensing Reconstruction by Adaptive PCA Sparse Basis and Nonlocal Similarity," Ksii Transactions on Internet and Information Systems, vol.8 no.8, pp.2851-2865, 2014. Article (CrossRef Link).

[7] Ashraf, R., Bashir K., Mahmood T., et al. "Content-based Image Retrieval by Exploring Bandletized Regions through Support Vector Machines," Journal of Information Science and Engineering, vol.32, pp.245-269, 2016.

[8] Ashraf, R., Ahmed, M., Jabbar, S. et al. "Content Based Image Retrieval by Using Color Descriptor and Discrete Wavelet Transform,” Journal of Medical Systems, vol.42, pp.42-44, 2018. Article (CrossRef Link).

[9] Takhar, D., Laska, J. N., Wakin, M. B., Duarte, M. F., Baron, D., Sarvotham, S., Kelly, K. F., and Baraniuk, R. G., "A new compressive imaging camera architecture using optical-domain compression," International Society for Optics and Photonics in Electronic Imaging, pp. 606509-606509, 2006. Article (CrossRef Link).

[10] Duarte, M.F., Davenport, M.A., Takhar, D., Laska, J.N., Sun, T., Kelly, K.F., and Baraniuk, R.G, “Single-pixel imaging via compressive sampling," IEEE Signal Processing Magazine, vol.25 no.2, pp. 83-91, 2008. Article (CrossRef Link).

[11] Shen Y., Li S., "Sparse Signals Recovery from Noisy Measurements by Orthogonal Matching Pursuit," Inverse Problems \& Imaging. vol. 9 no.1, pp.231-238, 2015. Article (CrossRef Link).

[12] Hale, E. T., Yin W., Zhang Y., et al. "Fixed-Point Continuation for $\mathrm{L}_{1}$-Minimization: Methodology and Convergence," Siam Journal on Optimization, vol.19 no.3, pp.1107-1130, 2008. Article (CrossRef Link).

[13] Yin, W., Osher, S., Goldfarb, D., and Darbon.J., "Bregman Iterative Algorithms for L1-Minimization with Applications to Compressed Sensing," SIAM Journal on Imaging Sciences, vol.1 no.1, pp. 143- 168, 2008. Article (CrossRef Link).

[14] Donoho, D. L., Tsaig, Y., Drori, I., and Starck, J.-L., "Sparse solution of underdetermined systems of linear equations by stagewise orthogonal matching pursuit," IEEE Transactions on Information Theory, vol. 58, no. 2, pp. 1094-1121, 2012. Article (CrossRef Link).

[15] Metzler, C. A., Maleki, A., and Baraniuk, R.G., "From denoising to compressed sensing," IEEE Transactions on Information Theory, vol. 62, no. 9, pp. 5117-5144, 2016. Article (CrossRef Link).

[16] Dong, W., Shi, G., Li, X., Ma, Y., and Huang, F., "Compressive sensing via nonlocal low-rank regularization," IEEE Transactions on Image Processing, vol. 23, no. 8, pp. 3618-3632, 2014. Article (CrossRef Link). 
[17] Figueiredo, M.A., Nowak, R.D., Wright S.J., et al. “Gradient Projection for Sparse Reconstruction: Application to Compressed Sensing and Other Inverse Problems, ” IEEE Journal of Selected Topics in Signal Processing, vol.1 no.4, pp.586-597, 2007. Article (CrossRef Link).

[18] Figueiredo, M. A., and Nowak, R. D., “An em algorithm for wavelet-based image restoration,” IEEE Transactions on Image Processing, vol. 12, no. 8, pp. 906-916, 2003. Article (CrossRef Link).

[19] Nowak, R. D. and Figueiredo, M. A., "Fast wavelet-based image deconvolution using the em algorithm," in Proc. of IEEE Conference Record of the Thirty-Fifth Asilomar Conference on Signals, Systems and Computers, vol. 1, pp. 371-375, 2001. Article (CrossRef Link).

[20] Li, C., Yin, W., Jiang, H., and Zhang, Y., “An efficient augmented lagrangian method with applications to total variation minimization," Computational Optimization and Applications, vol. 56, no. 3, pp. 507-530, 2013. Article (CrossRef Link).

[21] Wang, Y., Yin, W., and Zhang, Y., "A fast fixed-point algorithm for convex total variation regularization,” tech. rep., Working paper, 2007.

[22] Osher, S., Burger, M., Goldfarb, D., Xu, J., and Yin, W., “An iterative regularization method for total variation-based image restoration,” Multiscale Modeling \& Simulation, vol. 4, no. 2, pp. 460-489, 2005. Article (CrossRef Link).

[23] Cai, J. F., Osher, S., and Shen, Z., "Linearized bregman iterations for compressed sensing," Mathematics of Computation, vol. 78, no. 267, pp. 1515-1536, 2009. Article (CrossRef Link).

[24] Burger, M., Gilboa, G., Osher, S., Xu, J., et al., "Nonlinear inverse scale space methods," Communications in Mathematical Sciences, vol. 4, no. 1, pp. 179-212, 2006. Article (CrossRef Link).

[25] Osher, S., Mao, Y., Dong, B., and Yin, W., "Fast linearized bregman iteration for compressive sensing and sparse denoising," Communications in Mathematical Sciences, vol.8 no.1, pp.93-111, 2010. Article (CrossRef Link).

[26] Yin, W., Osher, S., Goldfarb, D., and Darbon, J., "Bregman iterative algorithms for L 1-minimization with applications to compressed sensing," SIAM Journal on Imaging Sciences, vol. 1, no. 1, pp. 143-168, 2008. Article (CrossRef Link).

[27] Huang, B., Ma, S., and Goldfarb, D., “Accelerated linearized bregman method, ” Journal of Scientific Computing, vol. 54, no. 2-3, pp. 428-453, 2013. Article (CrossRef Link).

[28] Burger, M., Gilboa, G., Osher, S., Xu, J., et al., "Nonlinear inverse scale space methods," Communications in Mathematical Sciences, vol. 4, no. 1, pp. 179-212, 2006. Article (CrossRef Link).

[29] Burger, M., Resmerita, E., and He, L., "Error estimation for bregman iterations and inverse scale space methods in image restoration,” Computing, vol. 81, no. 2-3, pp. 109-135, 2007. Article (CrossRef Link).

[30] Burger, M., Moller, M., Benning, M., and Osher, S., “An adaptive inverse scale space method for compressed sensing,” Mathematics of Computation, vol. 82, no. 281, pp. 269-299, 2013. Article (CrossRef Link).

[31] Ke, J. and Lam, E. Y., “Object reconstruction in block-based compressive imaging,” Optics express, vol. 20, no. 20, pp. 22102-22117, 2012. Article (CrossRef Link).

[32] Kerviche, R., Zhu, N., and Ashok, A., "Information-optimal scalable compressive imaging system,” Computational Optical Sensing and Imaging CM2D-2, 2014. Article (CrossRef Link).

[33] Mahalanobis, A., Shilling, R., Murphy, R., and Muise, R., "Recent results of medium wave infrared compressive sensing,” Applied optics, vol. 53, no. 34, pp. 8060-8070, 2014. Article (CrossRef Link).

[34] Wang, J., Gupta, M., and Sankaranarayanan, A. C., "Lisens-a scalable architecture for video compressive sensing," in Proc. of IEEE International Conference on Computational Photography, pp. 1-9, 2015. Article (CrossRef Link).

[35] Goyette, N., Jodoin, P., Porikli, F., Konrad, J., Ishwar, P., “Changedetection.net: a new change detection benchmark dataset," in Proc. of Proceedings of the IEEE Computer Vision Pattern Recognition Workshops (CVPRW), IEEE, Boston, pp. 1-8, 2012. 
[36] Foucart, S., "Hard thresholding pursuit: an algorithm for compressive sensing,” SIAM Journal on Numerical Analysis. Vol. 49, no. 6, pp.2543-2563, 2011. Article (CrossRef Link).

[37] Wang, Z., Bovik, A. C., Sheikh, H. R., and Simoncelli, E. P., "Image quality assessment: from error visibility to structural similarity," IEEE Transactions on Image Processing, vol. 13, no. 4, pp. 600-612, 2004. Article (CrossRef Link).

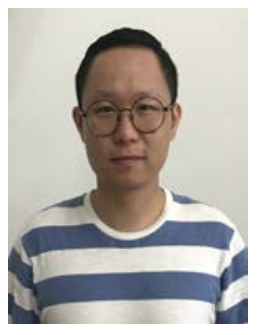

Yang Peng received the B.S. degree in Information System Engineering and M.S. degree in System Engineering from National University of Defense Technology, Changsha, Hunan, China, in 2008 and 2010, respectively, and received the PhD degree in System Engineering from National University of Defense Technology, Changsha, Hunan, China, in 2017. He is currently an assistant professor in the Department of System Engineering, National University of Defense Technology, Changsha, China. His current research interests include computational photography and deep learning in signal and image processing.

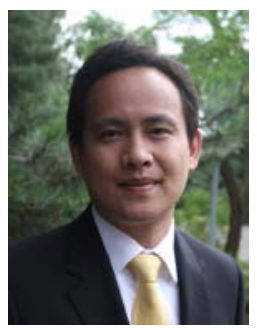

Yu Liu received his BS from Northwestern Polytechnical University, Xi'an, China in 2005. He received his MSc in image processing and $\mathrm{PhD}$ in computer graphics from the University of East Anglia, Norwich, United Kingdom, in 2007 and 2011, respectively. He is currently an associate professor in the Department of System Engineering, National University of Defense Technology. His research interests include image/video processing, computer graphics, and visual-haptic technology.

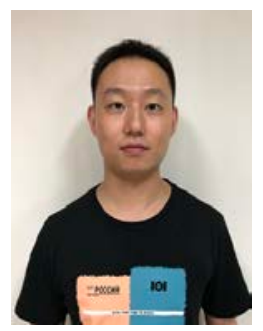

Kuiyan Lu received the master degree in Control Science and Engineering from National University of Defense Technology, Changsha, Hunan, China, in 2016. Currently, he is working on aerial vehicle panorama system in Shijiazhuang Flying College of PLAAF. His current research interests include panoramic imaging system and pattern recognition.

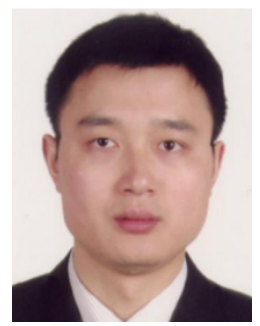

Maojun Zhang received his BS and PhD degrees in systems engineering from National the University of Defense Technology, Changsha, China, in 1992 and 1997, respectively. He is currently a professor in the Department of System Engineering, National University of Defense Technology. His research interests include computer vision, virtual reality, compuational photography. 